ORIGINAL ARTICLE

\title{
Diclofenac patch for topical treatment of acute impact injuries: a randomised, double blind, placebo controlled, multicentre study
}

H G Predel, R Koll, H Pabst, R Dieter, G Gallacchi, B Giannetti, M Bulitta, J L Heidecker, E A Mueller

Br J Sports Med 2004;38:318-323. doi: 10.1136/bjsm.2003.005017

\begin{abstract}
Objectives: To investigate the clinical efficacy and safety of a newly developed diclofenac patch in the topical treatment of blunt impact injuries.

Methods: This was a randomised, placebo controlled, double blind, multicentre study in 120 patients with traumatic blunt soft tissue injury. Within $3 \mathrm{~h}$ of the injury participants of sport competitions and training camps were enrolled and treated twice daily with the diclofenac or a placebo patch over a period of 7 days. Patients were randomised (1:1) to two parallel groups. Tenderness produced by pressure was measured twice daily during the first 3 days after enrolment as well as at day 7 . Tenderness was defined as the amount of pressure (measured by a calibrated caliper at the centre of the injury) that first produced a pain reaction as reported by the patient.

Results: The primary efficacy variable was the area under the curve for tenderness over the first 3 days. The diclofenac patch was significantly more effective than placebo $(p<0.0001)$. The treatment effect was $64.7 \mathrm{kp} \mathrm{h} / \mathrm{cm}^{2}$ (95\% confidence interval 48.7 to 80.9 ) between diclofenac and placebo patches. These results were supported by all secondary efficacy variables. The diclofenac patch produced rapid pain relief as reflected by the time to reach resolution of pain at the injured site which was significantly shorter compared to placebo $(p<0.0001)$. The diclofenac patch was well tolerated. The most frequently observed adverse events were local cutaneous adverse reactions (pruritus, rash) of minor severity occurring with the same frequency as in the placebo group.

Conclusions: A newly developed diclofenac patch is effective and safe for the treatment of blunt impact injuries.
\end{abstract}

See end of article for authors' affiliations

Dr J L Heidecker, Mepha

Ltd, Medical Department

Dornacherstrasse 114,

$\mathrm{CH}$ - 4147 Aesch,

Switzerland; janos.

heidecker@mepha.ch

Accepted 8 April 2003

$\mathrm{T}$ opical administration of non-steroidal anti-inflammatory drugs (NSAIDs) offers the advantage of local, enhanced drug delivery to affected tissues with a lower incidence of systemic adverse effects due to reduced plasma concentrations. ${ }^{2}$ The benefit of topical NSAID treatment has been proven in the past. ${ }^{1-4} \mathrm{~A}$ systematic review of 86 trials in 10160 patients showed that topical NSAIDs are effective compared to placebo in acute pain (number needed to treat [NNT] 3.9 to obtain good pain relief) and chronic pain conditions (NNT 3.1). ${ }^{3}$

Nevertheless, despite licensed status, there is scepticism that topical NSAIDs have any action other than as a rubefacient $^{5}$ and more randomised, placebo controlled studies were demanded. ${ }^{6}$

Different topical diclofenac formulations are available and are commonly used, particularly in Europe. Pharmacokinetic studies have shown that diclofenac, when applied topically, penetrates the skin barrier to reach joints, muscles, and synovial fluids in sufficiently high concentrations to exert local therapeutic activity. ${ }^{7-9}$ In contrast to conventional topical formulations such as creams or gels, plasters or patches permit a constant and continuous delivery of the active ingredient to the affected area by means of an occlusive bandage and slow release of the drug. ${ }^{10}{ }^{11}$ Studies have shown that after application of diclofenac hydroxyethylpyrrolidine (DHEP) plasters in patients with knee joint effusion, detectable diclofenac levels were measured in the sampled synovial fluid. ${ }^{10}$

Sport injuries require rapid treatment. Primary treatment with cold compresses is often supported by topical application of anti-inflammatory and anti-oedematous drugs. ${ }^{12}$ Optimal topical treatment should relieve acute pain and

reduce swelling in the injured area to restore normal movement. In addition, it should be safe and easy to handle.

A recently developed new diclofenac patch formulation, containing $140 \mathrm{mg}$ diclofenac sodium on an area of $140 \mathrm{~cm}^{2}$, might fulfil these criteria. Preclinical and clinical studies have shown good local tolerability and appropriate skin penetration characteristics thereby encouraging further investigations in patients. Despite the good skin penetration, diclofenac plasma levels were still lower by several orders of magnitude compared to standard oral treatment when administered twice daily for several days (unpublished data).

The objective of this trial was to determine the efficacy and tolerability of a newly developed diclofenac patch versus placebo in the treatment of acute sports impact injuries.

\section{METHODS}

The study was carried out at four centres in Germany as a randomised, placebo controlled, double blind clinical trial with two parallel treatments in adult male and female patients. The first patient was enrolled in February 2002 after approval of the study protocol had been obtained from independent ethics committees, and the study was completed in May 2002. All patients had given their informed consent before enrolment. The study was performed in accordance with the Declaration of Helsinki with all its amendments taking into account the principles of good clinical practice (GCP). Each patient was assigned in chronological order to one of the two treatment groups using a computer generated

Abbreviations: AUC, area under the curve; ITT, intention to treat; $\mathrm{kp}$, kilopond ( $1 \mathrm{kp}=9.80665 \mathrm{~N})$; NSAIDs, non-steroidal anti-inflammatory drugs; PP, per protocol; VAS, visual analogue scale 
block randomisation list (allocation ration 1:1; block size $=8$ ).

According to the study protocol, patients aged between 18 and 60 years suffering from fresh impact injuries (blunt, traumatic soft tissue injuries, and contusions) not requiring admittance to hospital were enrolled. The injuries occurred primarily during soccer, handball, and basketball competitions or during stays in related training camps. One of the investigators was present at these events and could therefore recruit the patients on the spot. The time between injury and treatment had to be less than $3 \mathrm{~h}$ without any pre-treatment (including previous cooling of the injured area). The tenderness reaction of the contralateral uninjured site had to be 1.5-fold higher than the value of the injured area with an absolute sensitivity to pain on the contralateral site of at least $2.5 \mathrm{kp} / \mathrm{cm}^{2}$. Pregnant women and women of child-bearing potential not using effective means of contraception were excluded, as well as patients with blood coagulation disorders and patients who had known allergies to diclofenac or to ingredients of the patch. In addition, patients who were too hairy at the injured area or who had skin disorders or open wounds in the area to be treated were excluded. Patients were instructed not to use any other preparations or dressings to treat the trauma. In particular, the use of NSAIDs, analgesics, or psychotropic agents was not allowed. Patients who took any pain relieving agents in the week before study start or who had consumed more alcohol than usual on the evening before enrolment were excluded.

The study medication (140 mg diclofenac sodium or placebo) was applied to the injured area twice daily for 7 days starting within $3 \mathrm{~h}$ of injury and immediately after enrolment. Any contact of the patch with humidity and water (for example shower or bath) had to be avoided. The percentage of used patches assessed at the end of treatment served as a measure of compliance. Moreover, during the first 3 days of treatment, one edge of the patch and the adjacent skin was marked with a stamp to confirm that the injured area was continuously covered with the patch.

An independent statistician produced the randomisation list for this clinical trial. Study medication was packaged according to this randomisation list by an independent contract research organisation (CRO). Nobody else had access to the randomisation list until the database was closed. Diclofenac patches (Olfen ${ }^{\circledR}$ Patch), each containing $140 \mathrm{mg}$ diclofenac sodium over a surface area of $140 \mathrm{~cm}^{2}$, and placebo patches were supplied by Mepha, Aesch, Switzerland. The placebo patch was visually indistinguishable from the active patch. The excipients of active and placebo patches were not identical, however, resulting in a different smell. To maintain blinding of the study, appropriate procedures were put in place as follows. Administration and removal of the study medication during the first 3 days were performed by a study nurse not involved in efficacy measurements (at day 3, the remaining study medication was handed out to the patients for self-application twice daily). Before all tonometric measurements, the nurse cleaned the injured area and the contralateral site with an oil- and alcohol-free tissue. Moreover, the tonometric readings were performed in a standardised and blinded fashion.

Medical and drug history as well as cause of the injury were assessed before study enrolment at the baseline examination. Physical examinations (including vital signs) as well as routine haematological (haemoglobin, red blood cells, platelets) and biochemical (sodium, potassium, alanine aminotransferase [ALAT], gamma glutamyl transpeptidase $[\gamma-\mathrm{GT}]$, creatinine) laboratory measurements were performed at baseline and at study end (day 7).
The tenderness reaction at the injury and contralateral site was measured with a tonometer twice daily during the first 3 days as well as at day 7. Between baseline measurement and first assessment after treatment initiation, there was a period of at least 6 but not more than $9 \mathrm{~h}$. The intensity of spontaneous pain on rest and movement were assessed at the same time and recorded in diaries the patients received at study start.

Tonometric measurements were performed using a calibrated caliper (Amitek Europe, Wuppertal, Germany; range $0-100 \mathrm{~N}$; surface $\mathrm{l} \mathrm{cm}^{2}$; with digital pressure recording). Increases in pressure during measurement were kept constant at $10 \mathrm{~N} / \mathrm{cm}^{2} / \mathrm{s}$. The site of measurement was marked with a water resistant marker, and measurements were repeated at the same site. The measurements were performed in such a way that the patient and the investigator could not read the actual pressure exerted (covered display). The device stored the last exerted pressure, which was then recorded. A tenderness reaction was defined as the pressure that, under the above conditions, produced a painful sensation. This method has been successfully applied in the past. ${ }^{13} 14$ Spontaneous pain sensation was recorded using a $10 \mathrm{~cm}$ visual analogue scale. ${ }^{15}$

The primary efficacy variable was the area under the curve (AUC), where the ordinate was the pressure that produced the first tenderness reaction, and the abscissa was the time after treatment (restricted to the first 3 days after first treatment).

Secondary efficacy variables were the per protocol (PP) analysis of the primary efficacy variable, the AUC of tenderness values over the whole study period ( 7 days), the AUCs over 3 and 7 days for the ratio of tenderness values injured/contralateral site, the time taken to reach the contralateral (healthy) value of tenderness (time to resolution of pain), the extent of spontaneous pain (on rest and movement) on a $10 \mathrm{~cm}$ visual analogue scale (VAS), and a global efficacy assessment by the patients and the investigators using a 4-point scale (excellent, good, moderate, and no effect).

Safety was evaluated on the basis of adverse events. In addition, patients and investigators assessed the tolerability of the patch under double blind conditions using a 4-point scale (excellent, good, fair, and poor) at the end of the study.

\section{Statistical analysis}

The AUC for tenderness over 3 days $\left(\mathrm{AUC}_{0-3 \mathrm{~d}}\right)$ after treatment start was determined as a summary measure for each patient using the cumulative trapezoidal rule. ${ }^{16}$ Seven hierarchical two-sided null hypotheses were stipulated in the study protocol. The first hypothesis $\left(\mathrm{AUC}_{0-3 \mathrm{~d}}\right.$ [diclofenac $]=\mathrm{AUC}_{0-3 \mathrm{~d}} \quad$ [placebo] $)$ was to be tested $(\alpha=5 \%)$. If this hypothesis was rejected, the second hypothesis was to be tested $(\alpha=5 \%)$; otherwise the confirmatory testing procedure stopped. If the second hypothesis was rejected, the third hypothesis was tested $(\alpha=5 \%)$; otherwise the confirmatory testing procedure stopped. This continued similarly up to the seventh hypothesis. The a priori ordering was as follows: AUC for tenderness over 3 days, time to resolution of pain, VAS pain assessment in motion, AUC of the ratio of tenderness values (injured/ contralateral site), AUC for tenderness over 7 days, global assessment of efficacy by investigator, and global assessment of efficacy by patient. Because of deviations from normal distribution, non-parametric Mann-Whitney-Wilcoxon tests were used for the comparison of treatment groups. This multiple testing procedure controls the multiple $\alpha$-level of $5 \% .{ }^{17}$ The treatment effect was estimated by means of Hodges-Lehmann estimators and by corresponding nonparametric $95 \%$ confidence intervals. ${ }^{18}$ 
The time needed to reach the contralateral value of tenderness was analysed by survival analysis methods (Kaplan-Meier estimator, two-sided log rank test). ${ }^{19}$

A sample size of 60 patients per group was determined statistically (assuming a standardised difference $\Delta=0.597$, a level of significance $\alpha=5 \%$, a power (1- $\beta)=90 \%$, and a twosided $t$ test situation) by CRMB Biometrics, Rheinbach, Germany. Each centre had to enrol at least eight but not more than 32 patients.

\section{RESULTS}

A total of 120 patients (60 per treatment group) were enrolled in the study by the four centres (range: 24-32 subjects per centre) at different sports competitions or in training camps. All patients randomised were treated with the study medication and included in the intention to treat analysis (ITT). No PP analysis was conducted because the number of patients in the PP population deviated less than 5\% from the ITT population, as prospectively defined in the statistical analysis plan. One patient in the diclofenac group stopped treatment after the efficacy measurements of day 3 due to an adverse event (local erythematous rash). This patient attended all subsequent assessments thereby fully complying with the ITT approach of the study. Thus, the ITT population and the population analysed for safety were identical in this clinical trial.

Demographic and clinical characteristics as well as baseline values of efficacy variables were well balanced between treatment groups (table 1). Mean time from injury to first treatment was comparable between groups (diclofenac, $47.4 \mathrm{~min}$; placebo, $48.3 \mathrm{~min}$ ). The distribution of the primary efficacy variable $\mathrm{AUC}_{0-3 \mathrm{~d}}$ showed no normal distribution. Hence, a non-parametric statistical analysis was performed. Table 2 presents a descriptive summary of the tenderness values and the AUC over 3 days. Treatment with the diclofenac patch was significantly more effective than placebo. Higher AUC values represent less pain sensitivity and thus a better clinical outcome. The results were consistent across all study centres. Similar results were obtained for the AUC over 7 days (table 2). Marked superiority of the active treatment was already proven at the first efficacy assessment (that is, $6 \mathrm{~h}$ after treatment initiation), which persisted until study end (fig 1). This was also reflected in the time needed to reach the value of the contralateral tenderness at the injured site (time to resolution of pain). Patients treated with the diclofenac patch recovered quickly with $73.3 \%$ of patients $(n=44)$ showing resolution of pain by day 7 compared to $6.7 \%$ of patients $(n=4)$ in the placebo group (fig 2; log rank test: two-sided $\mathrm{p}<0.0001$ ).

The average spontaneous pain measured on a visual analogue scale showed significant differences between active and placebo patches. In the diclofenac patch group, pain

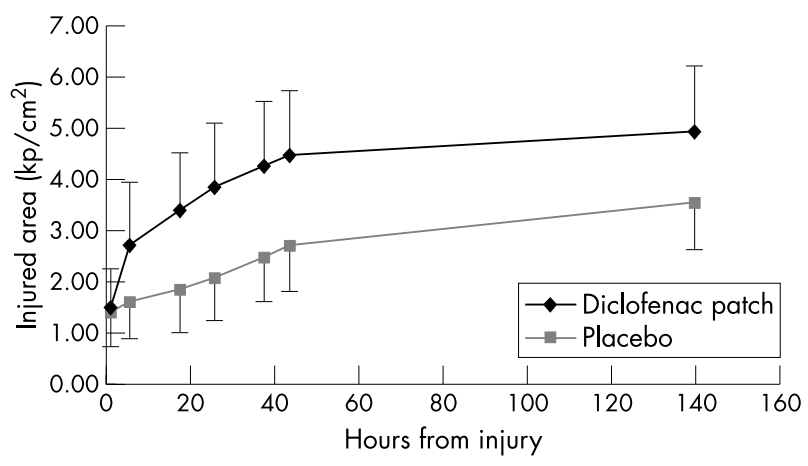

Figure 1 Tenderness reaction at injured site over time: intention to treat analysis. Values are means (standard deviations).

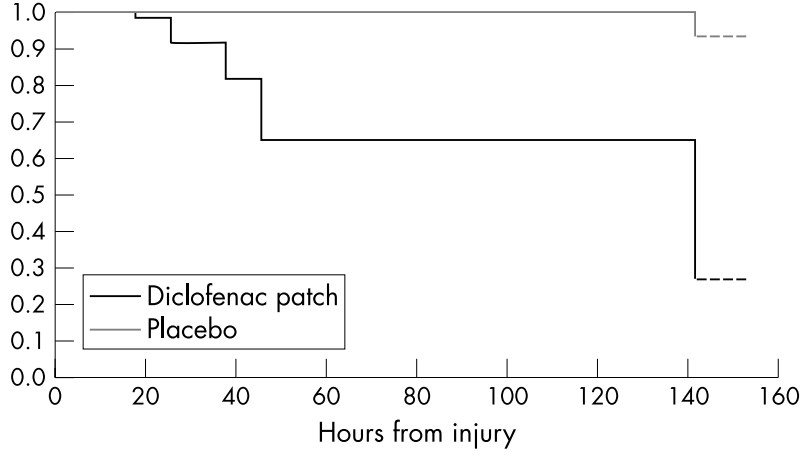

Figure 2 Kaplan-Meier survival curves for the time to resolution of pain: intention to treat analysis.

intensity was more effectively reduced than in the placebo group. This was observed for pain in motion (fig 3; $\mathrm{p}<0.0001)$ and pain at rest $(\mathrm{p}<0.0001)$.

Mean ratio of tenderness values (injured/contralateral site) improved faster in the diclofenac group compared to placebo (fig 4). AUCs of ratios over 3 and 7 days were higher in the diclofenac than in the placebo group $(32.4 v 20.1[\mathrm{p}<0.0001]$

Table 1 Basic characteristics of patients and baseline data (intention to treat)

\begin{tabular}{|c|c|c|}
\hline & $\begin{array}{l}\text { Diclofenac patch } \\
(n=60)\end{array}$ & $\begin{array}{l}\text { Placebo patch } \\
(n=60)\end{array}$ \\
\hline \multicolumn{3}{|l|}{ Sex } \\
\hline Male, $n(\%)$ & $38(63.3)$ & $35(58.3)$ \\
\hline Female, $n(\%)$ & $22(36.7)$ & $25(41.7)$ \\
\hline $\begin{array}{l}\text { Age (years) } \\
\text { Mean (median) }\end{array}$ & \multicolumn{2}{|c|}{ Age (years) } \\
\hline SD & 9.70 & 8.86 \\
\hline Q1; Q3 & $25 ; 37$ & $26 ; 37$ \\
\hline \multicolumn{3}{|l|}{ Height $(\mathrm{cm})$} \\
\hline Mean (median) & $177.2(178.0)$ & $176.2(174.5)$ \\
\hline SD & 8.20 & 10.30 \\
\hline Q1; Q3 & $171 ; 182$ & $168 ; 183$ \\
\hline \multicolumn{3}{|l|}{ Weight (kg) } \\
\hline Mean (median) & $71.3(72)$ & $71.6(69)$ \\
\hline SD & $12.03^{-1}$ & 14.06 \\
\hline Q1; Q3 & $62 ; 80$ & $64 ; 78$ \\
\hline \multicolumn{3}{|c|}{$\begin{array}{l}\text { Tenderness injured area } \\
\left(\mathrm{kp} / \mathrm{cm}^{2}\right)\end{array}$} \\
\hline Mean (median) & $1.5(1.3)$ & $1.4(1.3)$ \\
\hline SD & 0.77 & 0.67 \\
\hline Q1; Q3 & $1.0 ; 2.0$ & $0.9 ; 1.8$ \\
\hline \multicolumn{3}{|c|}{$\begin{array}{l}\text { Tenderness contralateral } \\
\text { area }\left(\mathrm{kp} / \mathrm{cm}^{2}\right)\end{array}$} \\
\hline Mean (median) & $5.1(4.8)$ & $4.7(4.4)$ \\
\hline SD & 1.43 & 1.39 \\
\hline Q1; Q3 & $4.0 ; 6.1$ & $3.8 ; 5.4$ \\
\hline \multicolumn{3}{|c|}{ Ratio of tenderness values* } \\
\hline Mean (median) & $0.28(0.28)$ & $0.29(0.29)$ \\
\hline SD & 0.08 & 0.09 \\
\hline Q1; Q3 & $0.23 ; 0.33$ & $0.23 ; 0.36$ \\
\hline \multicolumn{3}{|l|}{ VAS at rest (mm) } \\
\hline Mean (median) & $63(66)$ & $63(67)$ \\
\hline SD & 23.5 & 25.3 \\
\hline Q1; Q3 & $44 ; 81$ & $44 ; 85$ \\
\hline \multicolumn{3}{|c|}{ VAS in motion (mm) } \\
\hline Mean & $75(78)$ & $74(78)$ \\
\hline SD & 20.0 & 22.6 \\
\hline Q1; Q3 & $65 ; 92$ & $56 ; 95$ \\
\hline \multicolumn{3}{|c|}{ Location of injury, $\boldsymbol{n}(\%)$} \\
\hline Arm & $26(43.3)$ & $28(46.7)$ \\
\hline Leg & $31(51.7)$ & $30(50.0)$ \\
\hline Other & $3(5.0)$ & $2(3.3)$ \\
\hline
\end{tabular}


Table 2 Tenderness values $\left(\mathrm{kp} / \mathrm{cm}^{2}\right)$ and area under the curve (AUC) over 3 and 7 days $\left(\mathrm{kp} \mathrm{h} / \mathrm{cm}^{2}\right)$ at the injured site (intention to treat)

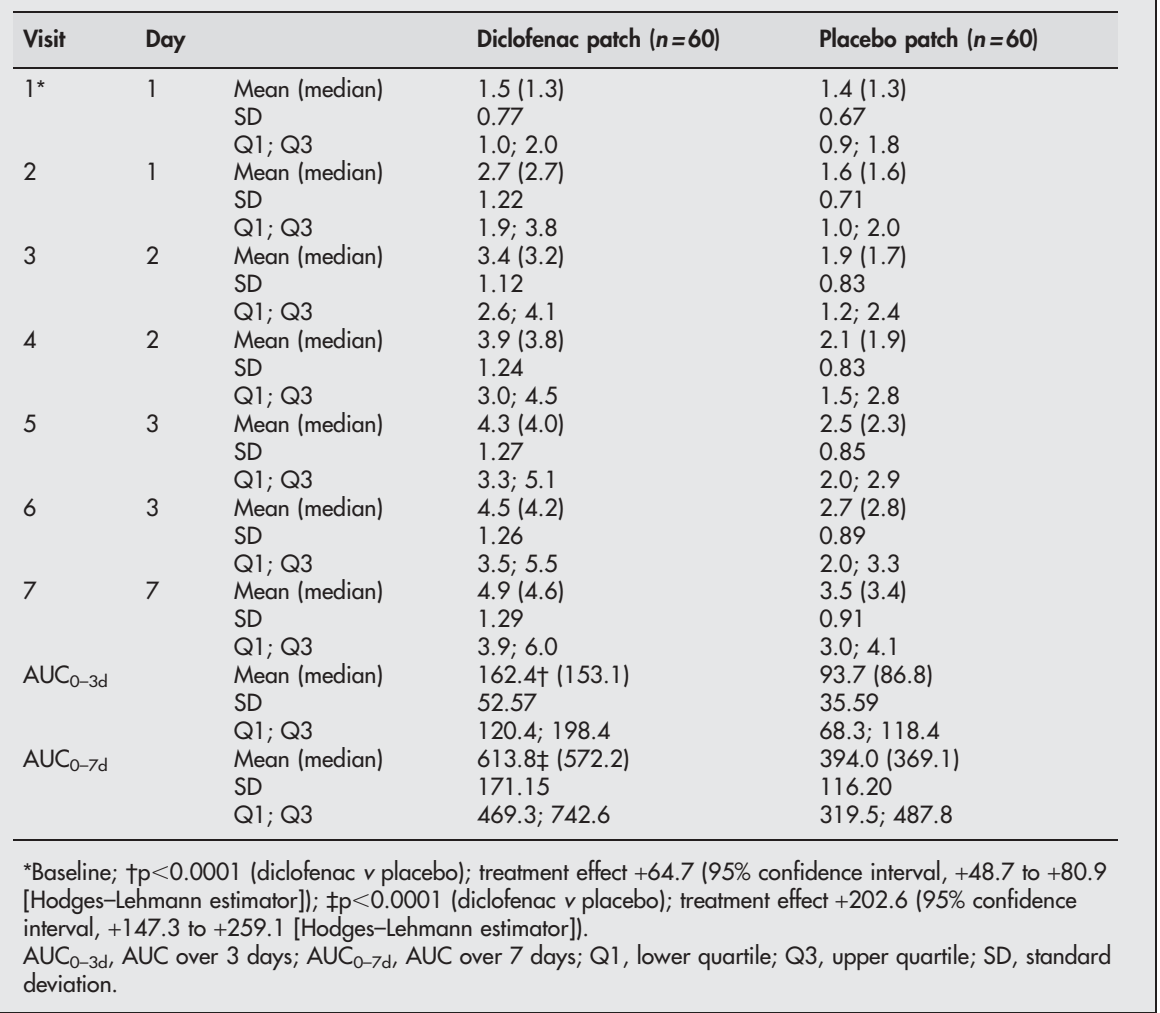

and $123.6 v 85.4[\mathrm{p}<0.0001]$, respectively). Higher values mean less pain.

In the overall assessment of efficacy, the investigators recorded significantly more patients with "excellent" or "good" efficacy in the diclofenac group than in the placebo group (diclofenac, 91.7\%; placebo, 8.3\%). The patient's assessment of efficacy revealed almost identical results with a $\mathrm{p}<0.0001$ for the comparison to placebo.

Overall, both treatments were well tolerated. A total of 12 patients $(10 \%)$ experienced 16 mild adverse events with no statistical or clinically relevant difference between treatment groups (table 3). All adverse events were rated as "possibly" or "certainly" related to study medication by the investigators, and all patients recovered without sequelae. The overall tolerability was assessed as "excellent" or "good" in nearly all cases by the investigators (diclofenac, 98\%; placebo, 95\%) and patients (diclofenac, $88 \%$; placebo, $85 \%$ ) (table 3 ).

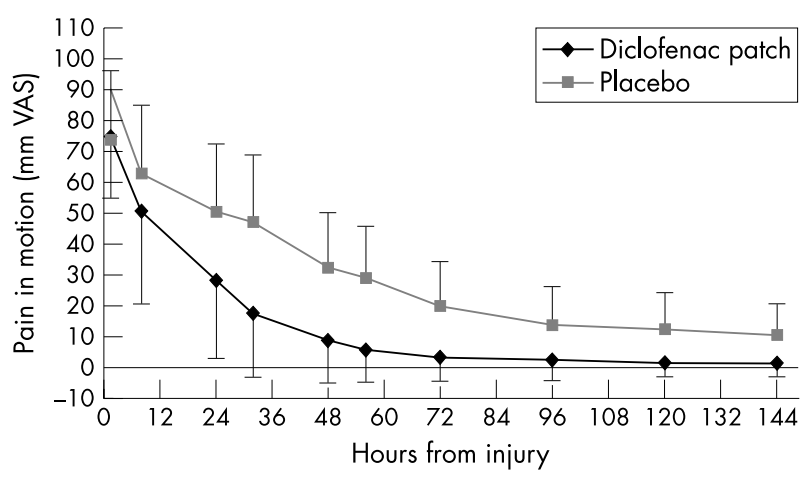

Figure 3 Pain in motion (visual analogue scale) over time: intention to treat analysis. Values are means (standard deviations).
Haematological and biochemical variables revealed no statistical or clinically meaningful differences between treatment groups during the course of the study. Likewise, no clinically relevant changes in vital signs were noted during the study.

\section{DISCUSSION}

The main outcome of this study was that a newly developed diclofenac patch is very effective and safe for the treatment of blunt impact injuries. Patients receiving the diclofenac patch had statistically and clinically significant reductions in pain scores and were free of pain significantly earlier than patients in the placebo group. The efficacy of the diclofenac patch was further supported by the "AUC of the ratios of tenderness values (injured $v$ contralateral site)". This variable was clearly superior for the group that received the diclofenac patch compared to the group that received the placebo patch. The

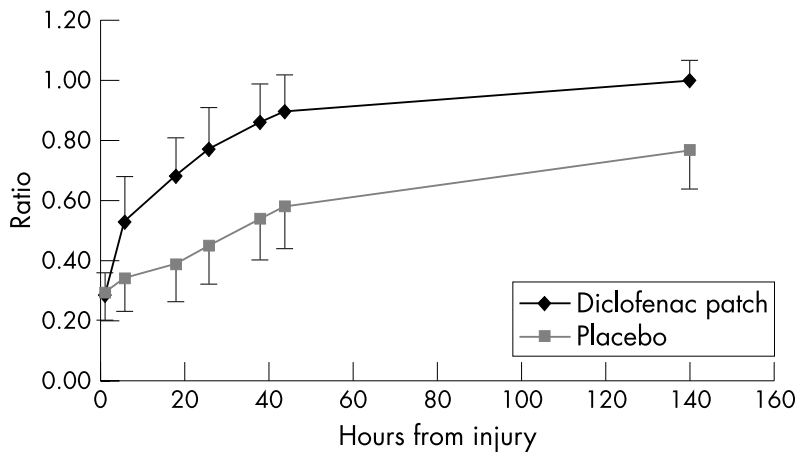

Figure 4 Ratio of tenderness values (injured/contralateral) over time: intention to treat analysis. Values are means (standard deviations). 
Table 3 Number (\%) of patients with adverse events and global tolerability assessments by investigators and patients (intention to treat)

\begin{tabular}{lcc}
\hline & Diclofenac patch $(\boldsymbol{n}=60)$ & Placebo patch $(\boldsymbol{n}=60)$ \\
\hline $\begin{array}{l}\text { Adverse event* (preferred term) } \\
\text { Pruritus }\end{array}$ & $2(3.3)$ & $2(3.3)$ \\
$\begin{array}{l}\text { Erythematous rash } \\
\text { Pustular rash }\end{array}$ & $3(5.0)$ & $7(11.7)$ \\
$\begin{array}{l}\text { Global assessment of tolerability by } \\
\text { investigators }\end{array}$ & $2(3.3)$ & \\
Excellent & $56(93.3)$ & $47(78.3)$ \\
Good & $3(5.0)$ & $10(16.7)$ \\
Fair & - & $2(3.3)$ \\
Poor & $1(1.7)$ & $1(1.7)$ \\
Global assessment of tolerability by patients & & - \\
Missing & $2(3.3)$ & $36(60.0)$ \\
Excellent & $44(73.3)$ & $15(25.0)$ \\
Good & $9(15.0)$ & $6(10.0)$ \\
Fair & $4(6.7)$ & $3(5.0)$ \\
Poor & $1(1.7)$ & \\
\hline${ }^{*}$ Multiple citations possible. & & \\
\hline
\end{tabular}

parameter takes additionally the subjective pain sensitivity into account. The favourable results were consistent across all efficacy endpoints. In terms of safety, the diclofenac patch was locally well tolerated and did not lead to systemic side effects. The adverse event profile was not different from placebo.

Sport injuries require rapid treatment. Primary treatment with cold compresses is often supported by topical application of anti-inflammatory and anti-oedematous drugs. ${ }^{12}$ An optimal topical treatment should relieve acute pain and reduce swelling in the injured area to restore normal movement. In addition, it should be safe and easy to handle. It was perceived that a recently developed new diclofenac patch formulation, containing $140 \mathrm{mg}$ diclofenac sodium over an area of $140 \mathrm{~cm}^{2}$, would be a viable treatment option for blunt soft tissue injuries. In contrast to conventional topical formulations such as creams or gels, patches or plasters permit a constant and continuous delivery of the active ingredient to the affected area by means of an occlusive bandage and controlled release of the drug. ${ }^{10}{ }^{11}$ Various studies suggested its usefulness in sport injuries ${ }^{20}$ as well as rheumatic ${ }^{21-23}$ and non-rheumatic diseases. ${ }^{24}$ Also other NSAIDs have meanwhile been formulated as patches or plasters. $^{25}$

Evaluation of efficacy of topical drugs in the acute treatment of blunt soft tissue injuries is complicated by the great variability in pain reaction and was often subject to failure. ${ }^{26}$ Pain is influenced by various factors such as the size and location of the injury, the outside temperature, general training status, and subjective sensitivity to pain. Hence, clinical studies must be highly standardised.

Pain assessment by visual analogue scale is frequently used in pain studies with topical preparations. It is obvious that this method has its limitations because of the subjectivity of the recordings, resulting in a large range of variation. These drawbacks can only partially be met by highly standardised measurement conditions and by using controlled, double blind study designs. A combination of a measurement method for induced (tonometric measurement) and spontaneous pain (assessment by VAS) seemed to be the most suitable way to accurately reflect the clinical situation of a blunt injury. Such an approach was selected in our study. The applicability and validity of tonometric measurements have been confirmed in the past. ${ }^{13}{ }^{14}$ The additional tonometric comparison with the uninjured contralateral area takes the subjective pain sensitivity into account by providing an "internal standard" and seemed therefore to be especially valuable for supporting the primary efficacy variable "AUC of tenderness values over 3 days" which is based on absolute values.

Only early treatment of an injury can usefully contribute to the relief of symptoms. Evaluation of a topical preparation for the acute treatment of blunt soft tissue injuries is certainly most informative if the application occurs very quickly after injury. Our study addressed this issue by recruiting patients at the actual site of the sports events to ensure standardised and timely treatment. Based on previous experience, a study duration of 7 days was thought to be sufficient to prove efficacy and local tolerability in blunt soft tissue injuries. ${ }^{13} 2728$

The presented study clearly shows that the newly developed diclofenac patch (Olfen ${ }^{\circledR}$ Patch) is effective for the treatment of blunt impact injuries. Patients receiving the diclofenac patch had statistically and clinically significant reductions in pain scores and were free of pain significantly earlier than patients in the placebo group. This allowed patients to continue their sports activities sooner thereby reducing the risk of additional injuries because pain free movement allows normal co-ordination. The efficacy results of this study support previous investigations in which the benefit of topical NSAID treatment has been proven. ${ }^{1-4}$

Pathophysiological mechanisms of sports impact injuries are similar to those of inflammatory reactions ${ }^{29}$ and such traumatically related inflammatory reactions of tendons, ligaments, muscles, and joints seem to be very similar in nature. ${ }^{30-32}$ Hence, the tested diclofenac patch might also be useful in conditions with the same underlying pathomechanism (for example, certain painful rheumatic diseases).

The diclofenac patch was well tolerated when administered twice daily over 1 week. Potential adverse reactions of topical NSAIDs can be divided into cutaneous reactions and systemic reactions. The latter were absent in our study which supports previous findings that the lower plasma concentrations achieved with topical NSAID administration is associated with reductions in systemic adverse effects. ${ }^{12}$ The most frequently observed adverse events in this trial were local cutaneous adverse reactions (pruritus, rash) of minor severity and occurring with the same frequency as in the placebo group. This is in line with previous studies with topical NSAIDs in which similar adverse reactions were observed. ${ }^{26}$ The number of adverse events was low considering that the patch situation represents a "semi-occlusive" environment, in which toxic or allergic effects might be amplified.

It can be concluded that the newly developed diclofenac patch (Olfen ${ }^{\circledR}$ Patch) is effective and safe in the treatment of blunt impact injuries. It complements the pharmaceutical 


\section{Take home message}

A newly developed diclofenac patch (Olfen ${ }^{\circledR}$ Patch) is effective and well tolerated in the treatment of blunt impact injuries. Patients treated with the diclofenac patch had significant reductions in pain scores and were free of pain significantly earlier compared to patients on placebo. The diclofenac patch might be used in indications with similar pathomechanisms

armamentarium for treating inflammatory reactions caused by sports impact injuries and might be used in indications with similar pathomechanisms.

\section{ACKNOWLEDGEMENTS}

Monitoring and statistical analysis of the study were carried out by the contract research organisations CRM Pharmaberatung GmbH and CRMB Biometrics GmbH, Rheinbach, Germany. The randomisation list was generated by Elisabeth Vieth, MSc, Cologne, Germany. Blinded study medication was prepared by CRO Wülfing Pharma $\mathrm{GmbH}$, Gronau/Leine, Germany.

\section{Authors' affiliations}

H G Predel, Deutsche Sporthochschule Cologne, Carl-Diem-Weg 6, Cologne, Germany

R Koll, Sports Medicine, Richard-Zanders-Str. 10, Bergisch Gladbach, Germany

H Pabst, Sports Medicine, Hirtenweg 2a, Grünwald, Germany

R Dieter, Sports Medicine, Fütererstr. 21, Munich, Germany

G Gallacchi, Schmerzklinik Kirschgarten, Hirschgässlein 11-15, Basle, Switzerland

B Giannetti, M Bulitta, CRM/CRMB GmbH, Rheinbach, Germany

J L Heidecker, Medical Department, Mepha Ltd, Aesch, Switzerland

E A Mueller, Clinical Pharmacology, Im Käppelefeld 6, Bad Krozingen, Germany

Mepha Ltd, Aesch, Switzerland provided the study medication and funded the study.

Conflict of interest: none declared.

\section{REFERENCES}

1 Heyneman CA, Lawless-Liday C, Wall GC. Oral versus topical NSAIDs in rheumatic diseases. A Comparison. Drugs 2000;60:555-74.

2 Green S, Buchbinder R, Barnsley L, et al. Non-steroidal anti-inflammatory drugs (NSAIDs) for treating lateral elbow pain in adults (Cochrane Review). In Cochrane Library. Issue 1. Oxford: Update Software, 2003. Date of most recent substantive amendment: 10 August 2001.

3 Moore RA, Tramer MR, Carroll D, et al. Quantitative systematic review of topically applied non-steroidal anti-inflammatory drugs. BMJ 1998;316:333-8.

4 Gotzsche PC. Non-steroidal anti-inflammatory drugs. Clinical review (extracts from 'Clinical Evidence'). BMJ 2000;320:1058-61.

5 Bateman DN, Kennedy JG. Non-steroidal anti-inflammatory drugs and elderly patients. The medicine may be worse than the disease. BMJ 1995;310:817-8.

6 Ogilvie-Harris DJ, Gilbart M. Treatment modalities for soft tissue injuries of the ankle: a critical review. Clin J Sport Med 1995;5:175-86.

7 Radermacher J, Jentsch D, Scholl MA, et al. Diclofenac concentrations in synovial fluids and plasma after cutaneous application in inflammatory and degenerative joint disease. Br J Clin Pharmacol 1991;31:537-41.
8 Davies NM, Anderson KE. Clinical pharmacokinetics of diclofenac. Clin Pharmacokinet 1997;33:184-213.

9 Müller M, Rastelli C, Ferri P, et al. Transdermal penetration of diclofenac after multiple epicutaneous administration. J Rheumatol 1998;25:1833-6.

10 Gallacchi G, Marcolongo R. Pharmacokinetics of diclofenac hydroxyethylpyrrolidine (DHEP) plasters in patients with monolateral knee joint effusion. Drugs Exp Clin Res 1993;19:95-7.

11 Assandri A, Canali S, Giachetti C. Local tolerability and pharmacokinetic profile of a new transdermal delivery system, diclofenac hydroxyethylpyrrolidine plaster. Drugs Exp Clin Res 1993;19:89-95

12 Heyneman CA. Topical nonsteroidal antiinflammatory drugs for acute soft tissue injuries. Ann Pharmacother 1995;29:780-2.

13 Koll R, Buhr M, Dieter R, et al. Wirksamkeit und Verträglichkeit von Beinwellwurzelextrakt (Extr. Rad. Symphyti) bei Sprunggelenksdistorsionen. Ergebnisse einer multizentrischen, randomisierten, placebo-kontrollierten Doppelblindstudie [Efficacy and safety of Extr. Rad. Symphyti in patients with ankle joint distortions. Results of a multi-centric, randomised, placebocontrolled, double blind study]. Z Phyłother 2000;21:127-34 (in German).

14 Wetzel D, Menke W, Dieter R, et al. Escin/diethylammonium salicylate/ heparin combination gels for the topical treatment of acute impact injuries: a randomised, double blind, placebo controlled, multicentre study. Br J Sports Med 2002;36:183-8.

15 Huskisson EC. Measurement of pain. Lancet 1972;2:1127-31

16 Gibaldi M, Perrier D. Pharmacokinetics. New York: Marcel Dekker, 1982.

17 Maurer W, Hothorn LA, Lehmacher W. Multiple comparisons in drug clinical trials and preclinical assays: a-priori ordered hypotheses. In: Vollmar J, ed. Biometrie in der chemisch-pharmazeutischen Industrie, Band 6. Stuttgart: Gustav Fischer Verlag, 1995:3-18.

18 Lehmann EL. Nonparametrics: statistical methods based on ranks. San Francisco: McGraw-Hill, 1975.

19 Matthews JNS, Altman DG, Campbell MJ. Analysis of serial measurements in medical research. BMJ 1990;300:230-5.

20 Jenoure $\mathbf{P}$, Segesser B, Lühti $U$, et al. A trial with diclofenac HEP plaster as topical treatment in minor sport injuries. Drugs Exp Clin Res 1993;19:129-37.

21 Dreiser RL, Tisne-Camus M. DHEP plasters as a topical treatment of knee osteoarthritis - a double-blind placebo-controlled study. Drugs Exp Clin Res 1993; 19:121-7.

22 Galeazzi M, Marcolongo R. A placebo-controlled study of efficacy and tolerability of a nonsteroidal anti-inflammatory drug, DHEP plaster, in inflammatory peri- and extra-articular rheumatological diseases. Drugs Exp Clin Res 1993;19:111-9.

23 Rosenthal M, Bahous I. A controlled clinical study on the new topical dosage form of DHEP plasters in patients suffering from localized inflammatory diseases. Drugs Exp Clin Res 1993;19:101-10.

24 Affaitati G, Vecchiet F, Lerza R, et al. Effects of topical diclofenac (DHEP plaster) on skin, subcutis and muscle pain thresholds in subjects without spontaneous pain. Drugs Exp Clin Res 2001;27:69-76.

25 Waikakul S, Danputipong P, Soparat K. Topical analgesics, indomethacin plaster and diclofenac emulgel for low back pain: a parallel study. J Med Assoc Thai 1996:79:486-90.

26 Vaile JH, Davis P. Topical NSAIDs for musculoskeletal conditions: a review of the literature. Drugs 1998;56:783-99.

27 Hess H, Groher W, Lenhart P, et al. Wirksamkeit und Verträglichkeit eines Kombinationsgels mit Aescin und Diethylamin-salicylat bei Patienten mit stumpfen Verletzungen [Efficacy and safety of an escin plus diethylamine salicylate combination gel in patients with sports impact injuries] Dtsch Z Sportmed 1996;47:423-30 (in German)

28 Pabst H, Segesser B, Bulitta M, et al. Efficacy and tolerability of escin/ diethylamine salicylate combination gels in patients with blunt injuries of the extremities. Int J Sports Med 2001;22:430-6.

29 Siegenthaler W. Klinische Pathophysiologie [Clinical pathophysiology]. 6th ed. Stuttgart: Georg Thieme Verlag, 1987 (in German).

30 Hungerberg W. Externa bei Sportverletzungen und rheumatischen Erkrankungen [Externa in sport injuries and rheumatic diseases]. Z Allg Med 1994; 70:322-5 (in German).

31 Almekinders LC. Anti-inflammatory treatment of muscular injuries in sport. Sports Med 1999:28:383-8.

32 Szczesny G, Olszewski L. The pathomechanism of posttraumatic edema of lower limbs: I. The effect of extravasated blood, bone marrow cells, and bacterial colonization on tissues, lymphatics, and lymph nodes. J Trauma 2002;52:315-22. 